also light loss, but when it is of normal length and brightness this is not the case. It is evident that we are dealing with two distinct conditions. All who have had practical acquaintance with the subject of colour-blindness are aware that all dichromics (so-called red-green blinds) are not equally colour blind. One dichromic will put a very full red and green together, but another will object to this, but will put together as a match a red and green occupying a relatively nearer position in the spectrum. The dichromic with the smallest neutral band in the centre of the spectrum, separating the two colours red and violet, has the best colour perception. This, it will be seen, is a prediction from the theory, and is inexplicable on any other. A dichromic with the smallest neutral band is very difficult to detect with any of the usual tests, and will generally pass them with ease. The reason of this is that a dichromic of this kind sees about six distinct differences in the spectrum; he sees green as a lighter and greger colour than red, and distinguishes between them just as a normal-sighted person distinguishes between bluish-greens and blues. This is how the colour-blind match wools. The dichromic are, therefore, those who see two true colours and grey. They regard red, orange, yellow, and half of the green as one colour; the other half of the green, blue, and violet as the other. The presence of a neutral band causes the colours corresponding to this portion of the spectrum to be seen as grey. Therefore the larger the neutral band the more colours will be classed as grey. If the rays which fall within one colour of the dichromic be mixed with those which fall within the other, grey will be the result. Therefore violet and red, instead of making a purple to the dichromic, make a grey, which is indistinguishable to them from the grey made by blue and green.

The next stage of evolution of the colour sense is when the colour-perceiving centre is sufficiently developed to distinguish three main colours in the spectrum. The third colour, green, appears in the centre of the spectrum -that is, at the third point of the greatest difference of refrangibility of the rays. In accurdance with the prediction of the theory, I found a considerable number of persons who saw the spectrum in this way, about 1.5 per cent. of men. Sir William R zmsay and Sir J. J. Thomson belong to this class which I have designated "trichromic." The trichromic see three main colours in the spectrum-red, green, and violet. They usually describe the spectrum as consisting of red, redgreen, green, green-violet, and violet. They do not see yellow and blue as distinct colours, and are therefore in continual diffisulty over them. There are very few of the tests in general use which can detect them, especially if names be not used. They will usually pass a matching test with ease. An examination with the spectrum shows that their colour perception is less than the normal in every part, though the curve has the same general shape. The three trichromics described in my recent paper on "Observations on Hue Perception" " each saw ten consecutive monochromatic patches in the spectrum instead of the 18 or 19 seen by those who see six colours in the spectrum. It is easy to show that the trichromic are dangerously colour-blind. They will mark out with the spectral apparatus a patch containing greenishyellow, yellow, and orange-yellow, and declare that it is absolutely monochromatic. When tested with coloured lights they find great difficulty with yellow and blue. Yellow is continually called red or green.

There are several other degrees of colour perception, and it may be well to say a word or two about them, though I class all above the trichromic with the normal-sighted for practical purposes, as they are not dangerously colour-blind and can always distinguish signal lights correctly. In the next stage of evolution four colours are seen in the spectrum, and the fourth colour appears at the fourth point of greatest difference of refrangibilitynamely, at the orange-yellow of the hexachromic or sixunit people; these persons I have designated " tetrachromic" because they see four distinct colours in the spectrum-that is, red, yellow, green, and violet. They do not see blue as a definite colour, and are continually classing blues with greens : they usually prefer to call blue, purplish-green. In the next stage in evolution there appeared those who see five colours in the spectrum-red, yellow, green, blue, and violet, blue being now recognised as a definite colour; these are the pentachromic group. These people pass all the tests in general use with ease; they, however, have a definitely diminished colour perception compared with the normal or those who see six colours in the spectrum. They mark out in the spectrum only 15 monochromatic patches instead of 18 They cannot see orange as a definite colour; for instance, they can never tell whether a strontium light, which is red, or a calcium light, which is orange, is being shown them. In the next stage of evolution orange is recognised as a definite colour, and thus we get the hexachromic or normal group, and, as we should theoretically expect, the yellow of the pentachromic is now split up into two colours-orange and yellow. The last stage of evolution which we appear to have reached are those who see seven colours in the spectrum, and the additional one is called indigo. These constitute the heptachromic group, and this seventh colour appears at the exact point which it should appear according to my theorynamely, between the blue and the violet. Persons belong. ing to this class have a marvellous colour perception and memory for colours. They will indicate a certain shade of colour in the spectrum, and then next day will be able to put the pointer at precisely the same point-a feat which is quite impossible to the ordinary normal-sighted person. They see a greater number of monochromatic patches in the spectrum than the hexachromic, but the curve has the same form. The marking out of the heptachromic does not appear correct to those who see six colours: for instance, the blue appears to invade the green and the indigo does not appear a definite colour at all. If, however, we bisect the blue of the seven-colour man and then bisect his indigo, on joining the centres we get the blue of the six-colour man, showing most definitely that the blue has been split up into two fresh colours.

It will be noticed that there is room for much further evolution, and we could go on splitting up the spectrum indefinitely it only we had the power to distinguish these finer differences, but, as a matter of fact, I have never met with a man who could see more than 29 monochromatio patches in the spectrum, and there are really millions, though by monochromatic patches I do not mean 29 separate colours. Time will not permit me to give an explanation in this paper of all the facts of vision and colour vision, accord. ing to this theory. I have dealt with the chief facts in my book on "Colour Blindness and Colour Perception," in the International Scientific Series, and various papers. I am not aware of any fact which does not support the theory. Hendon, N.W.

\section{A NOTE ON THE PATHOLOGY OF LEAD POISONING.}

BY KENNETH W. GOADBY, M.R.C.S. ENG., L.R.C.P. LOND., D.P.H. CANTAB.

PATHOLOGISY AND LECTURER ON BACTERIOLOGY, NATIONAL DEMTAL HOSPITAL;

AND

F. W. GOODBODY, M.D. Dub.,

ASSISTANT PROFESSOR OF PATHOLOGICAL GHEMISTRY, UNIVERSITY COLLEGE, LONDON.

THE symptoms of lead poisoning have been known for many years, and the literature relating to the subject is enormously large. Great as is the number of works, no initial stage of unanimity has been reached concerning the pathology. There are three conceivable channels by which lead may enter the system-namely : (a) through the gastro. intestinal canal; $(b)$ through the respiratory system; and ( $a$ ) through the skin. For many years the first of these channels was considered to be the one of primary importance, possibly owing to the prominence of colic as a symptom; but more recent investigation, particularly the statistical ones of His Majesty's Chief Inspector of Factories and the Chief Medical Inspector of Factories, have determined that the incidence of industrial lead poisoning varies directly as the dust produced in the particular lead industries. In 1905 one of us (K.W.G. ${ }^{1}$ ) instituted a series of experiments to test the possibility of producing lead poisoning in animals by causing them to inhale lead dust in the form of fine white lead dust obtained

1 Goadby, Kenneth W., Journal of Hygiene, vol. ix., April, 1909. 
from the exhaust ventilation air duct flues in a white lead works, such dust, for instance, as the workmen are exposed to during the packing of dry white lead. These experiments pointed very definitely to the lung rather than the stomach as the source of poisoning, animals fed with a gramme of lead carbonate per diem surviving those which inhaled 0.3 gramme every third day.

These preliminary experiments have been followed up by a further and extended series of experiments in which other compounds than white lead have been used for the investigations, and we have demonstrated that during the inhalation of finely divided lead compounds lead in the form of dust enters the lung, and becomes absorbed by the tissues of the lung, the lead being demonstrable in the alveoli of the lung, and at times in the protoplasm beyond, and that by lung absorption all the symptoms of lead-poisoning take place. The amount of lead dust present in the air in factories in which poisoning takes place amongst the workers is very much smaller than the quantity of lead present in the air of our inhalation cage. Thus Duckering ${ }^{2}$ estimates the amount of lead present in the total respired air of a man working in a tinning factory as about 0.014 gramme per diem. The amount of lead present in our cage air varied from 0.0001 gramme to 0.001 gramme per litre; and allowing for the respiration of the cat at about 50 cubic centimetres per respiration, and a respiration-rate of about 25 per minute, the cat would inhale 0.03 gramme of lead during the 30 minutes it was exposed in the cage, this being every other day, and occasionally with three-day intervals ; three months were required to produce unmistakable clinical symptoms. The quantity of lead found in the air of tinning factories is much larger than is the amount present in the air in the potteries, but it is not much in excess of the quantity present in dusty processes in white lead works. Our experimental conditions were certainly more severe than those of industrial processes. Still, from an experimental point of view it is of the greatest importance to induce the poisoning as rapidly as possible, providing always that the clinical symptoms of the animals are comparable to those of man under natural conditions. This we found to be the case. The animals suffered from emaciation which was progressive, paralysis, and in many instances died with symptoms suggestive of involvement of the brain. These symptoms are analogous and, we believe, homologous with the saturnine cachexia seen in lead workers, the wrist-drop and other forms of paralysis, and finally acute encephalopathy and lead mania.

Compounds of lead other than white lead were also used, and in every case symptoms similar to those observed in man have been produced. The other compounds of lead used were lead acetate, litharge, lead dust from de-silverising works, and lead bisilicate of a form used in the potteries. The animals used for experiment have been mainly cats (35), though one dog was used. These animals are especially susceptible to lead poisoning, and it is common knowledge that when kept as domestic pets in lead works they soon develop lead poisoning. In three instances we have ad ministered alcohol in addition to lead, and find that alcohol increases the susceptibility and diminishes the latent period of lead poisoning. Careful post-mortem examinations have been made of the animals that died. The tissues have been examined histologically with a view to determining the primary action of lead on the animal tissues and to establish the specific pathological lesions produced. We desire here to express our warm appreciation and grateful thanks to Dr. H. R. Clark, who has undertaken the histological examination of the nervous tissues and has also given us much valuable help and advice.

The material that comes into the hands of the patholngist in cases of industrial lead-poisoning is generally derived from chronic poisoning, rarely of acute poisoning, and it is particularly in these cases that a diagnosis of "death from lead poisoning" is an exceedingly difficult one to make. The literature, as has already been stated, is very extensive, and at the same time remarkably contradictory. Kobert, ${ }^{3}$ in summing up the general effect of lead on the animal body, states that "lead attacks especially the striped and unstriped muscles, the epithelium of the excretory glands, the neuroglia of the central nervous system, and it is especially a protoplasmic poison." The principal clinical manifestations of lead poisoning are lead colic, lead paralysis, and

Duckering, F., ibid., vol. viii., November, 1908 3 Rudolf Kobert: Lehrbuch der Intoxikationen, p. 361 perhaps lead encephalopathy. It is in the pathology of these symptoms that the greatest variation is to be found in the records of different workers.

One series of observers consider that the action of lead is directly upon the central nervous system, producing on the one hand colic and on the other paralysis of the motor nerves, as in the extensor paralysis of wrist drop (Quensel, ${ }^{4}$ Rudolf Meyer, ${ }^{5}$ Gesenius, ${ }^{6}$ \&c.). On the other hand, Küssmal and Paul Maier, ${ }^{7}$ Mosss $^{8}{ }^{8}$ and Galvini ${ }^{9}$ describe sclerosis of the solar plexus, cceliao ganglia, and also-particularly Eichorz ${ }^{10}$ - consider that the damage is one mainly located in the spinal cord. Another series of observers, among whom may be named Riegels, ${ }^{11}$ who examined 200 cases of colic, and Jaccoud and Weber, ${ }^{12}$ consider that such symptoms as colic, amaurosis, and amblyopia are referable to vaso-motor spasm of the blood-vessels, particularly in colic to the inferior mesenteric arteries, actual stasis producing the amaurosis. Galvini ${ }^{13}$ describes in a man of 54 who was subject to chronic saturninism and who died in a condition of extreme cachexia, perihepatitis, perisplenitis, wasting of the stomach, liver, and spleen, and inflammation of the solar plexus. Paul Maier ${ }^{14}$ found sclerosis of the connective tissues of the cœeliac plexus. Further, a later series of observers, amongst whom are Gravitz, ${ }^{15}$ Litten, ${ }^{16}$ Hamel, ${ }^{17}$ and Keil, ${ }^{18}$ describe a degeneration of the red blood corpuscles as an early symptom of lead poisoning. This consists of an alteration of the protoplasm of the blood discs with basophile staining. Some observers think that the peripheral nerves and the motor nerves undergo degeneration, as did Prevost and Binet. ${ }^{19}$ Nissl ${ }^{20}$ describes characteristic degeneration of the cortical cells of the brain. And, finally, Glibert, ${ }^{21}$ who fed a number of rabbits on lead carbonate in the form of pills, found congestion and stasis of the liver, fibroid changes and emphysema in the lungs, and kidney degeneration of a fibroid nature, and occasional microscopical hæmorrhage. Oliver ${ }^{22}$ notes kidney degeneration of a parenchymatous nature in rabbits poisoned with white lead, and Weber ${ }^{23}$ and Virchow ${ }^{24}$ describe degenerative nephritis. There is thus a considerable difference of opinion as to the exact action of lead, but distinct consensus of opinion that its pathological effects are widespread.

We think it will be possible to show by the histology of our animals that any one of the symptoms and histological findings described is consonant with the primary effects of lead intoxication-namely, microscopical hæmorrhages, affecting practically all the tissues of the body, as well as the nervous system, central and peripheral. A detailed account of our experiments is out of place here, and we have therefore summarised our results. The lead compounds were administered by inhalation, hypodermic injection, and feeding.

\section{Symptoms Produced.}

1. Loss of weight. - In all cases during the early stages of the experiments, in which the animals became ultimately poisoned, the weight at first increased, but subsequently fell progressively until the animal had lost one-third, sometimes two-thirds, of its body weight before it died. The control animals kept in the laboratory did not show this variation but maintained a steady level of weight. The animals fed on white lead have so far exhibited no signs of lead poisoning. and there is at the present time in the laboratory an anima

4 Quensel, F.: Archiv für Psychiatrie, Band xxxv., 1902

Meyer, Rudolf : Virchow's Archiv, Band xc., 1882 6 Gesenius, F. : Diss, Freiburg, 1887

1872 .

Kussmal and Paul Maier : Archiv für Klinische Medicin, Band ix.,

Mossé, M. : Zeitschrift für Klinische Medicin, Band 1., 1903. 9 Galvini, E.: Rivista Clinica, fas. 3, 1884.

11 Riegels. Korz: Virchow's Archiv, 3 ?, 120, 1890. 363. 1884.

Jaccoud and Weber: De l'Amaurose Saturnine, Thèse de Paris,

$$
\begin{aligned}
& 13 \text { Galvini, E.: loc. cit. } \\
& 14 \text { Mever, Rudolf : loc. cit. }
\end{aligned}
$$

15 Gravitz: Deutsche Medicinische Wochenschrift, 1899, No. 36 ; ibid. 1901, No. 52. 16 Ibid, 1899, No. 44

17 Hamel: Deutsches Archiv fur Klinische Medicin, Band Ixvii., 1900 18 Keil, R.: Archiv fúr Intern. Pharm, 10, 1902

19 Prevost and Binet: Revue Médicale de la Suisse Romande, II. 889.

20 Nissl. F.: Allgemeine Zeitschrift für Psychiatrie, Band xlv., 1892 ; and iv, 1897

2L Glibert, D. : Le Saturnisme Experimental, Extrait des Rapports Ann. de l' Insp. du Travail, 1906.

Oliver, Sir T. : Milroy Lectures, 1891, p. 96.

24 Virchow : Berliner Klinische Wochenschrift, 1884. 
which has been fed on white lead for eight months, at first with 0.1 gramme, then with 0.5 gramme, and finally 1.0 gramme of lead per diem. This animal even now exhibits no signs of poisoning. Three other animals fed on white lead, \&c., for shorter periods show no signs of poisoning.

2. Paraiysis. - The paralysis in our animals has been generally confined to the hind limbs. Those animals which have been inoculated with either acetate, carbonate, or bisilicate of lead died exhibiting signs of paralysis. The knee-jerks are at first increased and then become sluggish; considerable wasting of the muscles takes place; the animals tire upon the least exertion, a run of 24 yards completely exhausting an animal that is passing under the influence of lead. The animals also adopt voluntarily a characteristic attitude; when taken out of their cages the animals at first stand with their hind legs well extended, the position of the knee-joint being normal. In a few seconds the quadriceps extensor slowly yields and the animals finally crouch or sit down. At the post-mortem examination the muscle is wasted and flabby. When the animal rolls over on its side and attempts to rise afterwards there is considerable difficulty in extending the knee-joint, the animal getting up with a jerk of its other muscles. In advanced cases the animals are only able to jump three or four inches from the ground.

3. Mental condition. - The animals remained perfectly friendly until the paralysis became well established, and it was often difficult to listen to their hearts with a stethoscope on account of their persistent purring. Finally, a distinct mental change was noticed, the animals became torpid, then irritable, highly nervous, and developed well-marked intention tremor, all pointing to cerebral irritation. In three instances fits occurred immediately before death; in one case the fit was of an epileptiform nature, commencing with twitchings and ending in clonic spasm.

4. Eyes. - In two instances amaurosis was present. The reaction to accommodation and light is invariably sluggish or entirely lost; the retinal vessels were tortuous and hæmorrhage was seen in one instance. The loss of reaction to light and accommodation was noticed by one of us (K. W. G. $)^{25}$ in the first series of animals experimented upon.

5. Post-mortem appearanoes.-All the animals that died were submitted to an exhaustive necropsy. The post-mortem appearances were practically identical in all the animals: there was an entire loss of all subcutaneous and mesenteric fat, and the omentum was practically non-existent. Occasionally the diploë of the skull was deeply congested, more especially in the inoculated animals. The liver and intestines were injected, the liver especially being engorged with blood. The lungs as a rule were normal, but rather full of blood, and in the animals subjected to inhalation the bronchial glands were found enlarged and often full of blood. There was practically no fat around the kidneys, but the capsule of the kidneys stripped easily. In one or two instances shrinkage of the cortex was noticed, but the most prominent symptom was the marked injection of the vessels on the surface of the kidney. The kidney on section was engorged with blood. No extravasation of blood was seen in the peritoneal cavity, except in one instance where a hæmorrhage had occurred near the suprarenals and in the region of the kidney. Small punctiform hæmorrhages were at times found in the stomach, in the duodenum, and the rest of the intestine; they were more marked in the lower end of the intestine and particularly around the ileo-creal valve.

Further, in animals which had not received any lead by the mouth at all, and in which the poisoning was produced by the subcutaneous injection of a lead compound, there was very distinct blue-black staining of the upper half of the cxcum and extending up into the vermiform appendix. The spleen was normal. The muscles were normal but flabby; where paralysis had existed the corresponding muscle was found diminished in size. The surface of the brain appeared normal, but the vessels were somewhat larger than in a normal animal. The vessels of the spinal cord were also somewhat increased in size, but there were no obvious hæmorrhages, nor were any macroscopical hæmorrhages found on making sections of the brain.

6. Histological exumination.-Portions of the heart, lungs,

25 Goadby, Kenneth : loe. cit liver, spleen, intestines, stomach, and kidneys were preserved in formalin, cut in paraffin, and stained by Heidenheim's hæmatoxylin and eosin, and by van Geissen's method. Portions of the anterior crural nerve supplying the paralysed quadriceps extensors were examined in celloidin and also sections of the spinal cord and the cortex of the brain. This portion of the histology was undertaken by Dr. R. H. Olark. In all the tissues examined a distinct engorgement and in. crease in size of the blood-vessels were found; here and there the smaller vessels, particularly the capillaries, were over-distended, and in addition numbers of minute microscopical hæmorrhages were everywhere apparent in the brain, cord, liver, kidney and lung, \&c.

In the kidneys the same microscopical hæmorrhages were seen with some slight changes in the epithelium of the tubules and a small amount of parenchymatous inflammation, no doubt due to the hæmorrhages which had taken place. The kidney, on the whole, seemed to suffer less than the lungs or liver. And in this connexion it is worth noting that clinically no obvious signs of blood are noticeable in the urine of persons suffering from lead poisoning. Thickening of the arteries in the various organs was not well defined; the veins rather than the arteries appear to be the source of the hæmorrhages. In the anterior crural nerves supplying the paralysed quadriceps extensor minute hæmorrhages were found between the nerve bundles, but not generally invading the nerve bundles, nor was any degeneration found either in the nerves supplying the muscle or in the sections of the cord examined; in places it was seen that the hæmorrhage was producing pressure. Hæmorrhage was also found under the pia arachnoid, which was somewhat thickened.

The whole histological picture was one of microscopio hæmorrhage occurring in the various organs and tissues, and it is evident that the minute hæmorrhages are the precursors of the fibroid and sclerotic changes that pathologists have seen in the tissues derived from cases of lead poisoning. Moreover, it is important to note in this connexion that not a few of the symptoms in lead poisoning have been ascribed to stasis and vaso-motor spasm or vaso-constrictor spasm of the various blood-vessels. In poisoning by nickel carbonyl Armit ${ }^{26}$ has shown that the poisoning is due to the nickel and not to the CO, that the nickel is absorbed through the lung, and that injections of other nickel salts produce the same pathological changes as inhalation of nickel carbonyl. In animals poisoned by nickel minute hæmorrhages occur in all the tissues, especially the brain.

Further, and of great importance, Mott describes in a recent paper ${ }^{27}$ in the Archives of Neurology and Psychiatry the histology of a case of fatal lead encephalitis, in which he found minute hæmorrhages due to the breaking down of the cortical vessels of the brain, degenerative hyaline thickening of the walls of the small vessels, together with a hypertrophy of the branching glia cells. The case described by Mott was a coach-painter, who developed lead poisoning at his work. The clinical symptoms were progressive loss of power, epileptiform seizures, followed by a mental condition of restlessness and irritability, paralysis, tremor, fibrillary twitchings, impairment of vision, tortuosity of retinal vessels, all exactly conforming to the symptoms noted in our experimental animals. Histologically the heart, spleen, liver, and lung of Mott's case showed fibroid thickening with a condition of angio-sclerosis in which arteries and veins were involved; the head of the cæcum was darkly stained. The kidneys showed interstitial fibrosis and hyaline degeneration, while the vascular coils in the capsules were frequently replaced by a hyaline purple-staining mass. Microscopical hæmorrhage was found in the spinal cord ; the vessels showed thickening and hyaline degeneration. The staining of the lower part of the intestine, particularly in the region of the ileo-cæcal valve, is a point of considerable importance, as much of our work points to the excretion of lead by way of the freces, and in our animals which were poisoned by inoculation, lead was undoubtedly present in the large intestine, the staining of the lower part of the intestine being similar to that noted by Newton Pitt.

We conclude, therefore, from our experiments that the essential and primary action of lead intoxication is the

${ }^{3}$ Armit, H. W. : Journal of Hygiene, vol. vifi. ; Proceedings of the Royal Society, vol. Ixxvii., p. 420 .

27 Mott, F. W. : Archives of Neurology and Psychiatry, vol. iv., p. 117. 
production of minute and microscopical hæmorrhages in various portions of the body, including the nervous system. That the clinical symptoms of lead palsy, and its good prognosis when treated early, are explainable by the presence of minute hæmorrhages in the peripheral nerves. The presence' of these minute hæmorrhages in the nervous system also gives an explanation of the varied pathological findings of many previous workers.

\section{A STUDY OF SPINAL AN ESTHESIA IN CHILDREN AND INFANTS.}

From a series of 200 Cases.

By H. TYRRELL GRAY, M.A., M.C. CANTAB., F.R.C.S. ENG.,

RESIDENT MEDICAL SUPERINTENDENT, HOSPITAL FOR SICK CHILDREN, GREAT ORMOND-STREET, LONDON.

(Continued from p. 91\%.)

\section{II.-A STUdy OF THE SECOND SERIES OF 100 CASES.}

IT is apparent from a critical study of the first series of cases that in a considerable proportion of these there are certain disadvantages which, though not serious in themselves, may' detract from the utility of this method in the surgery of children. For instance, the occurrence of any of the symptoms recorded in the previous contribution in a case where serious collapse was present might add considerably to the critical condition of such patient and make it doubtful whether there is any advantage to be gained over general anæsthesia.

In the first series there was a boy about nine years of age suffering from streptococcal general peritonitis. The advisability of interference was considered, and, as general anæsthesia was out of the question on account of the extremely collapsed condition of the patient, it was decided to attempt operation under spinal anæsthesia. A small injection ( 3 centigrammes stovaine) was administered and the peritoneal cavity thoroughly cleansed, the whole operation lasting about 45 minutes; during this time there were no symptoms of shock and the gravity of the child's condition was in no way accentuated. There was no vomiting during the manipulation of the intestines, nor did any signs of toxæmia, pallor, sweating, \&c., supervene. The child died shortly after his return to the ward, or rather over an hour from the time of the injection. An interesting feature was the striking absence of shock during the manipulations within the abdomen, and, further, three points in the case present themselves for consideration as accounting for the death :-

1. The disease.-.The peritonitis was so advanced and general, and the patient so clearly toxic, that I think it possible death might have occurred within an hour or two if no operation had been attempted. It was clear, in any case, that the administration of general anæsthesia would not have permitted more than incision and drainage of the abdominal cavity, if as much.

2. The injeotion.-Against the view that the injection of stovaine accelerated death is the important fact that in all cases in this series where any symptoms were present, these supervened at the latest within 20 minutes from the administration of the anæsthetic. It is extremely unlikely, therefore, that the progress of the disease was accelerated by any of the toxæmic symptoms referred to, for these should have made themselves evident earlier, and the boy should have manifested serious symptoms during the progress of the operation.

3. On nо occasion in the first series did I succeed in obtaining anæsthesia of the abdomen of more than one hour's duration. I think it probable, therefore, that the shock of the manipulation made itself felt as the anæsthesia of the abdomen was passing off, and that it was this factor which added to the gravity of the collapse due to the disease and contributed to the fatal result.

Whichever of these views be accepted, however, it is clearly desirable to abolish or minimise, if possible, symptoms which, though they do not materially influence the course of routine operations, may add considerably to the existing collapse in "desperate" cases, where the use of spinal anæsthesia is so strongly indicated. The prevention of the occurrence of these symptoms is only possible if we have an accurate knowledge of their causation, and this I now propose to consider.

Mr. W. Peck, chemist to the Hospital for Sick Children, has during the last ten months been kind enough to spend a great deal of time and trouble in making all the chemical and physical observations I have asked of him-and his results, controlled and repeated, have been of great value. It has been estimated by Dr. J. Graham Forbes ${ }^{\mathrm{l}}$ that the normal amount of cerebro-spinal fluid in the body is about two ounces. He states that the solids average about 1 per cent. and include 0.8 to 0.9 salts and 0.1 to 0.2 proteid and extractives. It is the salts which are of the greatest importance in the subject under consideration, and these are approximately as follows:-Sodium chloride, 0.7 ; potassium chloride, 0.03 ; alkaline and earthy phosphates, 0.013 to 0.02 ; and sulphates and carbonates, traces. These figures are, of course, subject to considerable variation under different physiological conditions.

In a series of normal children, from whom I obtained cerebro-spinal fluid on the operating table, the specific gravity was found to vary from 1.0054 to 1.0071 , the fluid of older children tending to be of a higher density than that of infants and younger children. In extreme collapse, however, as would be anticipated, there is a considerable increase in the specific gravity of the cerebrospinal fluid. Thus in two infants with advanced intussusception the cerebro-spinal fluid was 1.0080 and 1.0076 specific gravity ; in another case the specific gravity rose to $1 \cdot 0083$. Since this variation is largely due to the crystalloid substances, the depression of the freezing point or the osmotic pressure of the cerebro-spinal fluid alters accordingly, and I hope to show that this is of some importance in connexion with the safety and utility of spinal anæsthesia. In the explanation of the unpleasant symptoms referred to authors have differed widely. Mr. A. E. Barker, ${ }^{2}$ for instance, attributes fainting and collapse to the fact that the paralysis of the abdominal muscles so far relieves intra-abdominal pressure that the large veins become engorged with venous blood, and so a cerebral anæmia is produced. From the experience in my own cases I have been unable to satisfy myself that this factor plays more than a very small part, if any, in the production of these symptoms and for the following reasons :-

1. These complications have not infrequently occurred in cases of low anæsthesia when there has been no paralysis of the abdominal muscles.

2. The abdominal muscles have frequently been completely paralysed without any untoward symptoms resulting.

3. In every laparotomy I have performed under spinal anæsthesia I have examined carefully for signs of venous engorgement but without success.

This last reason has led me also to disbelieve the further statement that in these cases symptoms are due to paralysis of the splanchnic nerves and the resulting arterial hyperæmia of the viscera, for I have always noted, and frequently demonstrated to others, the definite anæmia and pallor of the intestines during spinal anæsthesia.

4. If there were any marked tendency to engorgement of the mesenteric veins this factor would only be of importance in the erect position and should be counteracted (to a very great degree, at any rate) by the raised pelvis position employed by Mr. Barker or by the Trendelenburg position, adopted by many surgeons, especially on the continent. I have not found the former a controlling factor.

5. The onset of the symptoms takes place from 10 to 20 minutes after the injection of stovaine when the paralysis of the abdominal muscles has been present for some time (i.e., about 15 minutes). Further, the onset of pallor has been noted as being delayed as long as 25 to 30 minutes on occasion.

6. If Mr. Barker's explanation were the correct one, it would be expected that the vomiting would follow the pallor and the cerebral anæmia, but the reverse is the usual sequence.

I have been led to believe, therefore, that the etiology of these complications is to be sought for in another direction.

Now, in administering a spinal anæsthetic, fluid of a much higher specific gravity and a rapid diffusibility has hitherto been introduced into the spinal theca. Mr. Peck has

1 Graham Forbes: Fstratto dai Lavori e Riviste di Chimica e Microscopia Clinica, vol. i., Fasc. ii. and iii., October-November, 1908. 